\begin{tabular}{|c|l|}
\hline Title & Cell Chirality Induces Collective Cell Migration in Epithelial Sheets \\
\hline Author(s) & Sato, Katsuhiko; Hiraiwa, Tetsuya; Shibata, Tatsuo \\
\hline Citation & $\begin{array}{l}\text { Physical review letters, 115(18), 188102-1-188102-5 } \\
\text { https://doi.org/10.1103/PhysRevLett.115.188102 }\end{array}$ \\
\hline Issue Date & 2015-10-30 \\
\hline Doc URL & http://hdl.handle.net/2115/60271 \\
\hline Rights & @2015A merican Physical Society \\
\hline Type & article \\
\hline File Information & PhysRevLett.115.188102.pdf \\
\hline
\end{tabular}

Instructions for use 


\title{
Cell Chirality Induces Collective Cell Migration in Epithelial Sheets
}

\author{
Katsuhiko Sato, ${ }^{1,2, *}$ Tetsuya Hiraiwa, ${ }^{3,4}$ and Tatsuo Shibata ${ }^{1,5}$ \\ ${ }^{1}$ RIKEN Center for Developmental Biology, 2-2-3 Minatojima-minamimachi, Chuo-ku, Kobe 650-0047, Japan \\ ${ }^{2}$ Research Institute for Electronic Science, Hokkaido University, Sapporo 001-0020, Japan \\ ${ }^{3}$ Department of Physics, Freie Universität Berlin, 14195 Berlin, Germany \\ ${ }^{4}$ Department of Physics, Graduate School of Science, The University of Tokyo, Hongo, Tokyo 113-0033, Japan \\ ${ }^{5}$ RIKEN Quantitative Biology Center, Kobe 650-0047, Japan
}

(Received 25 December 2014; revised manuscript received 27 July 2015; published 27 October 2015)

\begin{abstract}
During early development, epithelial cells form a monolayer sheet and migrate in a uniform direction. Here, we address how this collective migration can occur without breaking the cell-to-cell attachments. Repeated contraction and expansion of the cell-to-cell interfaces enables the cells to rearrange their positions autonomously within the sheet. We show that when the interface tension is strengthened in a direction that is tilted from the body axis, cell rearrangements occur in such a way that unidirectional movement is induced. We use a vertex model to demonstrate that such anisotropic tension can generate the unidirectional motion of cell sheets. Our results suggest that cell chirality facilitates collective cell migration during tissue morphogenesis.
\end{abstract}

DOI: 10.1103/PhysRevLett.115.188102

PACS numbers: 87.19.ru, 87.17.Pq, 87.17.Rt

During embryonic development and wound healing, epithelial (cohesive) cells form a monolayer sheet and move in a unified direction. This phenomenon is known as collective cell migration [1-4]. The unidirectional motion of cohesive cells contributes to the morphogenesis of multicellular organisms, for example in the left-right symmetry breaking of organs $[5,6]$.

A notable feature of this collective movement is that adjacent cells maintain their attachments. These cell-cell connections are biologically important, because an embryo that is covered by epithelial sheets while undergoing morphological changes relies on their integrity to separate outside from inside. However, the attachments result in strong physical constraints to movement; i.e., they prevent the cells from easily extending protrusions as cells migrating individually can. In addition, even in sheets with no free leading edges, epithelial cells can migrate collectively $[5,6]$. The cell-cell attachments also tend to make the cells act as bipolar objects, because the left boundary of a cell is the right boundary of the adjacent cell, and the boundaries on both sides tend to have similar mechanical properties $[7,8]$. It remains unclear how these cohesive cells can undergo unidirectional motion without a leading edge while maintaining cell-cell adhesion.

In contrast to such migration mechanisms of cell sheets, the mechanisms by which cell sheets are deformed are relatively well understood. A typical example of this is germ-band elongation in the fly [9]. In this phenomenon, myosin molecules are localized at the cell boundaries that are perpendicular to the anterior-posterior (AP) axis; this is due to cell polarity in the plane parallel to the cell sheet, which is called "planar polarity" [10,11]. The relatively high concentration of myosin generates strong contraction, which shrinks the cell boundaries, and the cells undergo autonomous intercalation along the dorsal-ventral axis $[9,12]$. The polarized contraction also plays an important role in the bending of cell sheets $[13,14]$.

In this way, the contractions and planar polarity of epithelial cells contribute to morphological changes in cell sheets, and presumably influence collective migration. Although much progress has been made in understanding the collective motion of cells [15], the associations between properties at the cellular level and movement at the tissue level remain unclear.

Here we provide a simple possible mechanism for the collective migration of epithelial cells. In this model, the strength of the contraction of cell boundaries depends on their orientation with respect to a fixed direction, and the direction in which the contraction is largest is tilted with respect to the fixed direction. We use a vertex model to demonstrate that the combination of direction-dependent contraction and spatial variation of the cell properties, such as the strength of adhesion between cells, along a given direction leads to unidirectional movement of the cells in a direction perpendicular to the given direction. The basis of this movement is repeated junction remodeling between cells arising from chirally polarized contraction at cell boundaries.

Let us consider a simple cell sheet in two dimensions as depicted in Fig. 1, in which the cells are confined by two parallel lines located at $y= \pm L_{y} / 2$; these lines represent the boundaries between the cell sheet and the other tissues. We impose periodic boundary conditions at $x= \pm L_{x} / 2$ and ask whether these epithelial cells can collectively, perpetually, and deterministically move in the positive $x$ direction, driven only by mechanical interactions between the cells. Here "collectively" means that either the entire cell sheet or a cell group within the sheet moves, and 


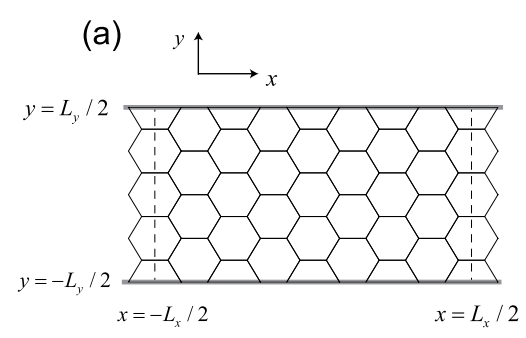

(b)

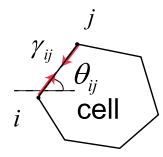

(c) $\mathrm{T} 1$ transition

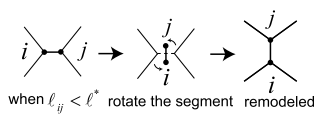

FIG. 1 (color online). Setup of the model. (a) The cell sheet is sandwiched between two parallel lines fixed at $y= \pm L_{y} / 2$. These represent the boundaries between other tissues and the cell sheet under consideration. Periodic boundary conditions are imposed at $x= \pm L_{x} / 2$. (b) A contraction force $\gamma_{i j}$ acting on the cell boundary $i j$ depends on the angle $\theta_{i j}$ between the cell boundary and the positive $x$ axis. (c) If the length of some cell boundary $i j$ becomes shorter than a given small value $\ell^{*}$, the boundary is rotated $90^{\circ}$ and the connection is remodeled (T1 transition).

"deterministically" means that the direction of the movement does not depend on the initial cell configuration or the intensity of any noise.

To address this question, we start with symmetry arguments for a particle model, because, as shown below, the vertex model that we consider herein is equivalent to the particle system. The dynamical equations of the particle model are given by

$$
\frac{d \boldsymbol{r}_{i}}{d t}=\boldsymbol{f}_{i}\left(\left\{\boldsymbol{r}_{j}\right\}, t\right)
$$

where $\boldsymbol{r}_{i}=\left(x_{i}, y_{i}\right)$ is the position vector of the $i$ th particle, $\boldsymbol{f}_{i}=\left(f_{i}, g_{i}\right)$ is a set of smooth functions of time $t$ and the positions of the particles, $\left\{\boldsymbol{r}_{j}\right\}=\left\{\boldsymbol{r}_{1}, \boldsymbol{r}_{2}, \ldots, \boldsymbol{r}_{N}\right\}$, where $N$ is the total number of particles. First we decompose the right-hand side of Eq. (1) into even and odd functions with respect to the $x$ and $y$ components of $\left\{\boldsymbol{r}_{j}\right\}$ as

$$
\frac{d \boldsymbol{r}_{i}}{d t}=\boldsymbol{f}_{i}^{(++)}+\boldsymbol{f}_{i}^{(+-)}+\boldsymbol{f}_{i}^{(-+)}+\boldsymbol{f}_{i}^{(--)} .
$$

The explicit forms of these are $\boldsymbol{f}_{i}^{(I J)} \equiv \Pi_{x}^{(I)} \Pi_{y}^{(J)} \boldsymbol{f}_{i}$ with $\Pi_{\alpha}^{( \pm)} \equiv\left(1 \pm P_{\alpha}\right) / 2$, where $I$ and $J$ take the sign + or - , and $\alpha$ takes $x$ or $y . P_{\alpha}$ is a linear operator acting on vector functions of $t$ and $\left\{\boldsymbol{r}_{j}\right\}$, defined as $P_{\alpha} \boldsymbol{g}\left(\left\{\boldsymbol{r}_{j}\right\}, t\right) \equiv$ $R_{\alpha} \boldsymbol{g}\left(\left\{R_{\alpha} \boldsymbol{r}_{j}\right\}, t\right)$ for any $\boldsymbol{g}$. $R_{\alpha}$ is the reflection operator, i.e., $\quad R_{x}(a, b)=(-a, b) \quad$ and $\quad R_{y}(a, b)=(a,-b)$ for any $(a, b)$.

Note that $\boldsymbol{f}_{i}^{(++)}$is invariant under the three transformations, (i) $(x, y) \rightarrow(-x, y)$, (ii) $(x, y) \rightarrow(x,-y)$, and (iii) $(x, y) \rightarrow(-x,-y)$. Here "invariant" means that the time evolution equations do not change form under the transformation. Similarly $\boldsymbol{f}_{i}^{(+-)}$is invariant only under (i), $\boldsymbol{f}_{i}^{(-+)}$is invariant only under (ii), and $\boldsymbol{f}_{i}^{(--)}$is invariant only under (iii). We call $\boldsymbol{f}_{i}^{(++)}, \boldsymbol{f}_{i}^{(+-)}$, and $\boldsymbol{f}_{i}^{(-+)}$, the isotropic, $y$-asymmetric, and $x$-asymmetric terms, respectively. If $\boldsymbol{f}_{i}$ involves an explicit driving force, such as $\boldsymbol{f}_{i}=(a, 0)$ $(a>0)$, it is included in the $x$ asymmetric term, $\boldsymbol{f}_{i}^{(-+)}$. We do not consider explicit driving forces in this article, and thus, in this symmetry argument, we will not further consider $\boldsymbol{f}_{i}^{(-+)}$. We refer to $\boldsymbol{f}_{i}^{(--)}$as the chiral term, because the mirror image of a system with $f_{i}^{(-)}$cannot be transformed to its original state by a rotation, much like for a helix of definite handedness. An example of a system containing $\boldsymbol{f}_{i}^{(--)}$is the case where each particle pulls other particles situated to its upper right and lower left, while it pushes particles situated to its upper left and lower right.

For this system to move collectively in the positive $x$ direction, the chiral term $f_{i}^{(--)}$is necessary, because the time-evolution equations must change under $(x, y) \rightarrow(-x, y)$. At the same time, the $y$ asymmetry term $f_{i}^{(+-)}$is also necessary, otherwise the system is the same after rotation by $180^{\circ}$, which would not result in unidirectional motion. In other words, in the absence of $x$ asymmetric terms, both the $y$ asymmetry term $\boldsymbol{f}_{i}^{(+-)}$and the chiral term $f_{i}^{(--)}$are necessary, and the combination of these terms may give rise to a directional collective movement.

Next, we apply the above-mentioned symmetry argument to the question of collective cell migration of epithelial cell sheets. To describe the dynamics of the sheet, we use a vertex model that describes the forces in each cell and the possible rearrangement of the cells [16-18]. In this model, cohesive cells are represented by polygons. The configuration of cells in the sheet is completely determined by the positions of the vertices of the polygons, $\left\{\boldsymbol{r}_{j}\right\}=\left\{\boldsymbol{r}_{1}, \boldsymbol{r}_{2}, \ldots, \boldsymbol{r}_{N}\right\}$, where $\boldsymbol{r}_{i}$ is the position vector of the $i$ th vertex and $N$ is the number of vertices, and the connections between the vertices. Mechanical forces in the cells are expressed by the derivatives of a potential function $E$, the form of which is

$$
\begin{aligned}
E\left(\left\{\boldsymbol{r}_{j}\right\},\left\{\gamma_{k l}\right\}\right)= & \frac{K}{2} \sum_{\alpha=1}^{M}\left(A_{\alpha}-A_{0}\right)^{2}+\sum_{\langle i j\rangle} \gamma_{i j}(t) \ell_{i j} \\
& +\frac{K_{p}}{2} \sum_{\alpha=1}^{M}\left(L_{\alpha}-L_{0}\right)^{2}
\end{aligned}
$$

where $A_{\alpha}$ and $L_{\alpha}$ are the area and perimeter of the $\alpha$ th cell, respectively, and $A_{0}$ and $L_{0}$ are its preferred constants, respectively. $M$ is the total number of cells. $\ell_{i j}$ is the length of the cell boundary $i j$, which connects the $i$ th and $j$ th vertices, $\gamma_{i j}$ is the line tension acting on the boundary $i j$, and $\langle i j\rangle$ means that the indices run over all the cell boundaries. The first term in Eq. (3) represents the hydrostatic pressure acting on the cell boundaries. The bulk 
modulus $K$ is set to be constant throughout this paper $(K=4)$. The second term represents the contraction forces acting on the cell boundaries; these are due to the actomyosin cables beneath the plasma membrane. The third term represents the tendency for the cell perimeter to be conserved. Some cells prefer a round shape $[8,19]$, so we impose the relation $L_{0}=2 \sqrt{\pi A_{0}}$ so that as $K_{p}$ increases, the shape of the cell tends to become rounded. In our model not only $\left\{\boldsymbol{r}_{j}\right\}$ but also $\left\{\gamma_{i j}\right\}$ evolve over time.

The time evolution equation for $\boldsymbol{r}_{i}$ is obtained by balancing the forces at the $i$ th vertex [16],

$$
-\eta_{i} \frac{d \boldsymbol{r}_{i}}{d t}-\frac{\partial E\left(\left\{\boldsymbol{r}_{j}\right\},\left\{\gamma_{k l}\right\}\right)}{\partial \boldsymbol{r}_{i}}=0,
$$

where $-\partial E / \partial \boldsymbol{r}_{i}$ is the net force acting on the vertex, and $-\eta_{i} d \boldsymbol{r}_{i} / d t$ is the friction force that represents the vertex's resistance to change of position, and the friction constant $\eta_{i}$ represents the strength of the attachments between the cells. In our model, $\eta_{i}$ depends on the vertex index $i$. Remodeling of boundaries is implemented in the same way as reported elsewhere [16,17] [see Fig. 1(c) for a brief explanation].

The time-evolution rules for $\gamma_{i j}$ are given as follows. Experimental observations show that $\gamma_{i j}$ fluctuates in time $[8,20]$. Thus, we first decompose $\gamma_{i j}$ into its mean value, $\bar{\gamma}_{i j}$, and the remaining part, $\xi_{i j}$, as

$$
\gamma_{i j}(t)=\bar{\gamma}_{i j}(t)+\xi_{i j}(t),
$$

and assume that $\xi_{i j}$ is colored Gaussian noise that satisfies $\left\langle\xi_{i j}(t)\right\rangle=0$ and $\left\langle\xi_{i j}\left(t_{1}\right) \xi_{k l}\left(t_{2}\right)\right\rangle=\delta_{i k} \delta_{j l} b^{2} \exp \left(-\left|t_{1}-t_{2}\right| / \tau_{1}\right)$, where the intensity $b$ and correlation time $\tau_{1}$ of the noise are non-negative constants. $\delta_{i j}$ is the Kronecker delta. Experimental observations also show that $\bar{\gamma}_{i j}$ depends on the orientation of the cell boundary $i j[9,13,21]$. Since the characteristic time for myosin to relocate (several tens of seconds) is much shorter than that of cell configuration change (several tens of minutes), we set up $\bar{\gamma}_{i j}$ to be a given function $\hat{\gamma}$ of the angle $\theta_{i j}$ between the cell boundary $i j$ and the $x$ axis at the time $t$, i.e.,

$$
\bar{\gamma}_{i j}(t) \simeq \hat{\gamma}\left(\theta_{i j}(t)\right) .
$$

Since cohesive cells tend to be bipolar, we determine the form of $\hat{\gamma}$ as

$$
\hat{\gamma}\left(\theta_{i j}\right)=\gamma_{0}+\gamma_{1} \cos 2\left(\theta_{i j}-\theta_{0}\right),
$$

where $\gamma_{0}$ and $\gamma_{1}$ are positive constants, and $\theta_{0} \in[0, \pi)$ is the angle at which the line tension reaches a maximum. By appropriately choosing $\theta_{0}$, we can express the planar polarity of the cells. For example, in germ-band elongation, $\theta_{0}=\pi / 2$ for all cell boundaries if the $x$ axis coincides with the AP axis. In our model, $\theta_{0}$ varies between cells and depends on the center of each cell's boundary.
With the above-mentioned values, Eqs. (5) through (7), and noting that $\theta_{i j}$ is a function of $\left\{\boldsymbol{r}_{j}\right\}$, Eq. (4) is closed in terms of $\left\{\boldsymbol{r}_{j}\right\}$, i.e.,

$$
\frac{d \boldsymbol{r}_{i}}{d t}=-\left.\frac{1}{\eta_{i}} \frac{\partial E\left(\left\{\boldsymbol{r}_{j}\right\},\left\{\gamma_{k l}\right\}\right)}{\partial \boldsymbol{r}_{i}}\right|_{\gamma_{k l}=\hat{\gamma}\left(\theta_{k l}\right)+\xi_{k l}} .
$$

This is the equation that we consider in this article. The boundary conditions at $y= \pm L_{y} / 2$ are obtained by adding to $E$ in Eq. (3) the potential given by $E_{B}=$ $K_{B}\left[\sum_{i \text { on upper boundary }}\left(y_{i}-L_{y} / 2\right)^{2}+\sum_{i \text { on lower boundary }}\left(y_{i}+\right.\right.$ $\left.L_{y} / 2\right)^{2}$ ], where $K_{B}$ is a large constant. To solve Eq. (8) numerically, we used the Euler method with an appropriate time step.

Notably, the time-evolution equation for the vertex model, Eq. (8), generally consists of isotropic terms. For example, if we set $\eta_{i}=\eta_{0}$ (const) for all $i$, and $\gamma_{1}=0$ or $\theta_{0}=0$ or $\pi / 2$, all the terms on the right-hand side of Eq. (8) become isotropic. However, if we set $\gamma_{1}>0$ and $\theta_{0} \neq 0$ nor $\pi / 2$, then chiral terms arise. In addition, if we make the parameters $\eta_{i}$ and $\theta_{0}$ depend on the $y$ coordinate, then $y$ asymmetric terms emerge in Eq. (8). The symmetry arguments shown above suggest that the latter situation, which contains both chiral and $y$ asymmetric terms, can result in unidirectional motion of the cell sheet.

First, we examine the case in which only chiral terms are introduced into the system; i.e., $\eta_{i}=1.0$ for all $i, \gamma_{1}=0.5$, and $\theta_{0}=\pi / 4$ for all cell boundaries. To make it easier to see the behavior of the system, we reduce the total number of cells, as shown in Fig. 2(a). For a large number of cells, the final results are qualitatively the same. As $\theta_{0}=\pi / 4$, the cell boundaries inclined to the right tend to shrink due to their relatively strong contraction forces, while the cell boundaries inclined to the left tend to expand due to their relatively weak contraction forces. With this situation, at some time point, such as $t=3.7$ in Fig. 2(a), four cells $(1,10,7$, and 4$)$ meet at a single point due to the shrinkage (a)

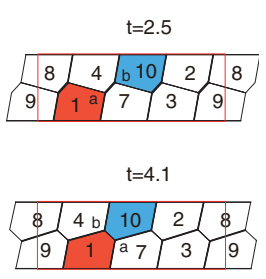

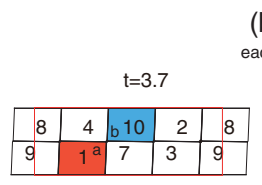

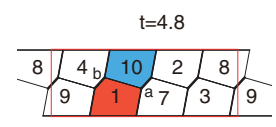

(b)

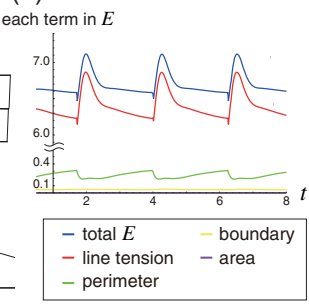

FIG. 2 (color online). Spontaneous and continuing shear movement of epithelial cells due to directionally dependent contractions; $\theta_{0}=\pi / 4$. (a) Snapshots of cell configurations. (b) Time series of each term in $E$. The total value of $E$ can increase due to the directional dependence of $\gamma_{i j}$ [see Eq. (9)]. The parameter values are $\gamma_{0}=0.5, \gamma_{1}=0.5, K=4.0, K_{p}=1.0, A_{0}=0.35$, $L_{y}=1, \quad L_{x}=8 \sqrt{3} / 5, \quad b=0, \quad \eta_{i}=1.0, \quad \ell^{*}=0.001, \quad$ and $K_{B}=100.0$. The units of all parameters are arbitrary. 
of cell boundary $a b$, and this causes a junction remodeling (T1 transition). After the junction remodeling, the cell boundary $a b$ becomes the boundary between cells 1 and 10 , and its direction is altered from $\theta_{a b} \simeq \pi / 4$ to $\theta_{a b} \simeq 3 \pi / 4$. This cell boundary $a b$ then tends to lengthen. These changes in the cell boundaries at $t=2.5$ result in the configuration seen at $t=4.8$, in which the upper cells are shifted to the left by one cell. This sliding motion of the cells is maintained as long as the cell polarity (directional dependence of $\gamma_{i j}$ ) persists (see Supplemental Material [22], Movie 1 for details).

This model results in continued movement while the existing vertex models approach an equilibrium state with decreasing $E[16,18]$ because here, $\gamma_{i j}$ obeys a different time-evolution rule than that followed by $\boldsymbol{r}_{i}$. In mathematical terms, for $b=0$ (no noise)

$$
\begin{aligned}
\frac{d E}{d t} & =\sum_{i=1}^{N} \dot{\boldsymbol{r}}_{i} \frac{\partial E}{\partial \boldsymbol{r}_{i}}+\sum_{\langle i j\rangle} \dot{\gamma}_{i j} \frac{\partial E}{\partial \gamma_{i j}} \\
& =-\sum_{i=1}^{N} \frac{1}{\eta_{i}}\left(\frac{\partial E}{\partial \boldsymbol{r}_{i}}\right)^{2}-\sum_{\langle i j\rangle} \sum_{k=1}^{N} \frac{1}{\eta_{k}} \frac{\partial E}{\partial \boldsymbol{r}_{k}} \frac{\partial \hat{\gamma}_{i j}}{\partial \boldsymbol{r}_{k}} \frac{\partial E}{\partial \gamma_{i j}} .
\end{aligned}
$$

Although the first term on the right-hand side, which represents the dissipation of $E$ caused by friction, is always nonpositive, the second term, which represents the effect of the change in $\gamma_{i j}$, can be positive and increase $E$ [see Fig. 2(b)].

Next we consider the case in which both chiral and $y$ asymmetric terms are introduced. To do this, we change $\eta_{i}$ along the $y$ axis; i.e., the vertices on the upper boundary at $y=L_{y} / 2$ have a high friction constant $\eta_{i}=100.0$ and the other vertices have the usual one $\eta_{i}=1.0$. In this case, although the upper cells hardly move, the lower cells move to the right (see Supplemental Material [22], Movie 2). That is, unidirectional movement of the cell sheet is achieved. Note that the direction of movement does not depend on the initial cell configuration but is determined only by $\theta_{0}$ and $\eta_{i}$. In another case, where $\theta_{0}=\pi / 4$ for $\left(y_{i}+y_{j}\right) / 2>0$, $\theta_{0}=3 \pi / 4$ for $\left(y_{i}+y_{j}\right) / 2<0, \eta_{i}=100.0$ for vertices on the boundaries at $y= \pm L_{y} / 2$, and $\eta_{i}=1.0$ for the other vertices, only the center parts of the cell sheet move to the right; this result resembles collective cell movement of the lateral line in zebrafish [23] [see Fig. 3 and Supplemental Material [22], Movie 3].

With this model, we also investigated the average speed of a cell sheet, defined as $\bar{v}:=\left(\int_{0}^{\tau_{2}} d t \sum_{i=1}^{N} d x_{i} / d t\right) / N \tau_{2}$, for a long interval $\tau_{2}$, as a function of the parameters of the cell [see Fig. 4]. Numerical simulations revealed the following. (i) In general, fluctuations in $\gamma_{i j}$ increase $\bar{v}$ [Fig. 4(d)], which is due to the fact that noise facilitates cell rearrangements. (ii) The movement is most efficient when $\theta_{0}=\pi / 4$ (or $3 \pi / 4$ ) [Fig. 4(b)]. (iii) $K_{p}$ must be appropriately sized for movement to occur [Fig. 4(c)]; for $K_{p} \gg 1$, the cell

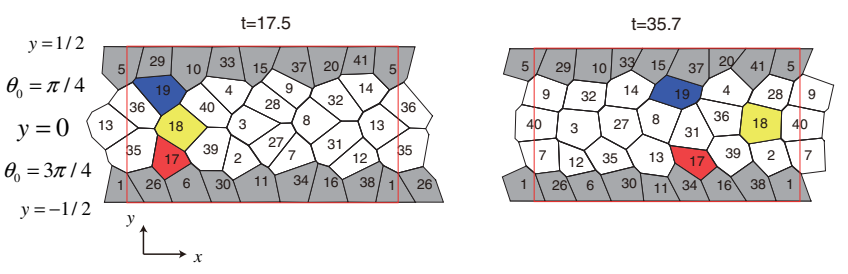

FIG. 3 (color online). Unidirectional movement of a cell sheet due to directional dependent contraction and spatial variation of $\theta_{0}$ along the $y$ axis. Cells are colored so that the changes in configuration can be easily seen. The parameters are $\tau_{1}=1.0$, $\gamma_{0}=0.2, \gamma_{1}=0.2, K=4.0, K_{p}=1.0, A_{0}=0.048, L_{y}=1$, $L_{x}=\sqrt{3}, K_{B}=100.0, \ell^{*}=0.001, b=0.045, \theta_{0}=\pi / 4$ for $\left(y_{i}+y_{j}\right) / 2>0$ and $\theta_{0}=3 \pi / 4$ for $\left(y_{i}+y_{j}\right) / 2<0$, and $\eta_{i}=$ 100.0 for vertex $i$ on the boundaries at $y= \pm L_{y} / 2$ and $\eta_{i}=1.0$ for the other vertices. The units of all parameters are arbitrary.

boundaries barely shrink, because the cells tend to remain round. For $K_{p} \ll 1$, the tensile force in the $\theta_{0}$ direction dominates. At each vertex, the forces that act in this direction are balanced. Consequently, along this direction, the distances between the vertices are maintained; thus, junction remodeling barely occurs. This result suggests that the cells should be appropriately rounded in order to move collectively. (iv) When there is no noise $(b=0)$, in general, $\bar{v}$ depends on the initial cell configuration; i.e., some hysteresis appears in $\bar{v}$ [blue curves in Figs. 4(a) and 4(c)]. If we add noise to the system $(b>0)$, the hysteresis is removed. Our model allows other system parameters to be varied, such as the width of the cell sheet, and a comparison can then be made with experimental

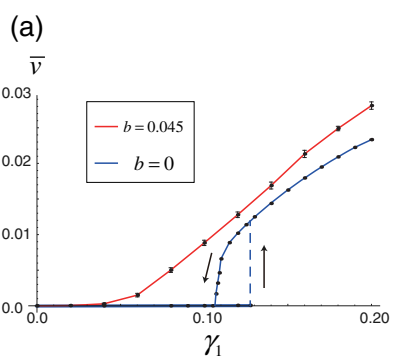

(c)

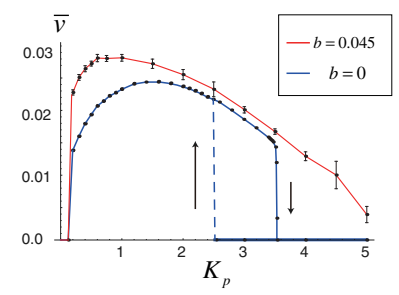

(b)

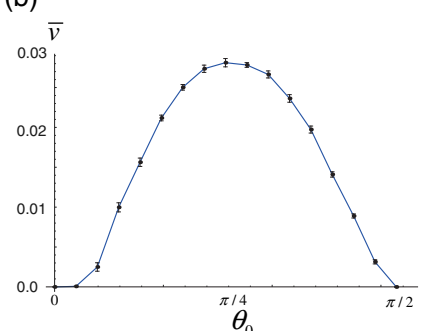

(d)

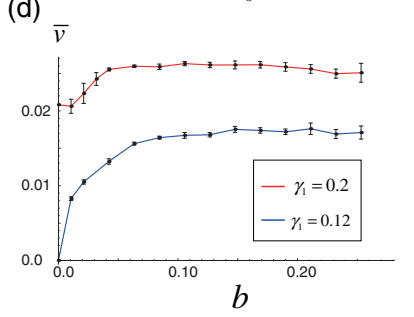

FIG. 4 (color online). Average speed of the collective movement of cells as a function of the various parameters: (a) $\gamma_{1}$, (b) $\theta_{0}$, (c) $K_{p}$, and (d) noise intensity $b$. Error bars indicate the standard deviation of $\bar{v}$ (sample size $=10$ ). The parameter values used here are the same as those used in Fig. 3 except where indicated in each graph. 
results [24]. A detailed qualitative study of our model will be presented elsewhere.

We have demonstrated that a direction-dependent tension, which is strengthened in the direction $\theta_{0}$ inclined from the major axis of the system, induces spontaneous and continued shear movement in a cell sheet. When such anisotropic tension is combined with the spatial dependence of $\eta_{i}$ or $\theta_{0}$, it leads to unidirectional movement of the system. We note that this movement results from local processes; i.e., each cell is just trying to shrink the boundaries at the upper left and lower right and expand those at the upper right and lower left when $0<\theta_{0}<\pi / 2$. Therefore, we can say that an intrinsic chiral property of cells drives the directional motion of the cell sheets.

Chiral behavior of cells has been reported elsewhere recently [25-27] and has been linked to chirally asymmetric dynamics of parts of the cytoskeleton, such as the actomyosin cortex beneath cell-surface membranes $[28,29]$. More recently, chiral mechanical torque generation in cells has been reported in C. elegans, which contributes to the formation of the left-right body axis [30]. At the tissue scale, the shape of cells and the distribution of adhesion molecules along cell boundaries showed left-right asymmetry during the process of forming embryonic hindgut twists in Drosophila [31,32]. In a recent collaborative study [33] we found that left-right asymmetric remodeling of cell boundaries can drive the collective cell movement involved in the genital disc rotation of Drosophila [5,6]. The observed relationship between the directions of cell chirality and cell movement is in agreement with the findings of the current article. A similar mechanism might also contribute to other types of collective cell migration [1,23]. Our scenario in which cell chirality plays a role in collective cell movement can be tested experimentally by evaluating the directional bias in the extension and contraction of cell boundaries.

We thank S. Yabunaka, M. Nishikawa, Y. Ishimoto, S. Okuda, H. Yi, W. K. Kim, and E. Kuranaga for valuable comments and discussions. We also thank H. Hiraga for critical reading of the manuscript. This work was supported by a Grant-in-Aid for Scientific Research from the Japan Society for the Promotion of Science (K. S. and T. S.) and by the Alexander von Humboldt Foundation (T. H.).

*Corresponding author.

katsuhiko_sato@es.hokudai.ac.jp

[1] P. Friedl and D. Gilmour, Nat. Rev. Mol. Cell Biol. 10, 445 (2009).

[2] D. J. Montell, Nat. Rev. Mol. Cell Biol. 4, 13 (2003).

[3] V. Lecaudey1, G. C.-Akdogan, W. H. J. Norton, and D. Gilmour, Development 135, 2695 (2008).

[4] G. Trichas, B. Joyce, L. A. Crompton, V. Wilkins, M. Clements, M. Tada, T. A. Rodriguez, and S. Srinivas, PLoS Biol. 9, e1001019 (2011).
[5] M. Suzanne, A. G. Petzoldt, P. Speder, J.-B. Coutelis, H. Steller, and S. Noselli, Curr. Biol. 20, 1773 (2010).

[6] E. Kuranaga, T. Matsunuma, H. Kanuka, K. Takemoto, A. Koto, K.-I. Kimura, and M. Miura, Development 138, 1493 (2011).

[7] J. Davies, Mechanisms of Morphogenesis (Academic, New York, 2005).

[8] B. Aigouy, R. Farhadifar, D. B. Staple, A. Sagner, J. C. Roper, F. Julicher, and S. Eaton, Cell 142, 773 (2010).

[9] C. Bertet, L. Sulak, and T. Lecuit, Nature (London) 429, 667 (2004).

[10] J. A. Zallen, Cell 129, 1051 (2007).

[11] F. Pilot and T. Lecuit, Dev. Dyn. 232, 685 (2005).

[12] J. P. Trinkaus, Cells into Organs: The Forces that Shape the Embryo (Prentice-Hall, Englewood Cliffs, NJ, 1984).

[13] T. Nishimura, H. Honda, and M. Takeichi, Cell 149, 1084 (2012).

[14] J. M. Sawyer, J. R. Harrell, G. Shemer, J. Sullivan-Brown, M. Roh-Johnson, and B. Goldstein, Dev. Biol. 341, 5 (2010).

[15] T. Vicsek and A. Zafeiris, Phys. Rep. 517, 71 (2012).

[16] T. Nagai and H. Honda, Philos. Mag. B 81, 699 (2001).

[17] A. G. Fletcher, J. M. Osborne, P. K. Maini, and D. J. Gavaghan, Prog. Biophys. Molec. Biol. 113, 299 (2013).

[18] R. Farhadifar, J.-C. Roper, B. Aigouy, S. Eaton, and F. Julicher, Curr. Biol. 17, 2095 (2007).

[19] A. K. Classen, K. I. Anderson, E. Marois, and S. Eaton, Dev. Cell 9, 805 (2005).

[20] A. C. Martin, M. Kaschube, and E. F. Wieschaus, Nature (London) 457, 495 (2009).

[21] M. Rauzi, P. Verant, T. Lecuit, and P.-F. Lenne, Nat. Cell Biol. 10, 1401 (2008).

[22] See Supplemental Material at http://link.aps.org/ supplemental/10.1103/PhysRevLett.115.188102 for movies that describe how our model moves.

[23] P. Haas and D. Gilmour, Dev. Cell 10, 673 (2006).

[24] S. R. Vedula, M. C. Leong, T. L. Lai, P. Hersen, A. J. Kabla, C. T. Lim, and B. Ladoux, Proc. Natl. Acad. Sci. U.S.A. 109, 12974 (2012).

[25] J. Xu, A. Van Keymeulen, N. M. Wakida, P. Carlton, M. W. Berns, and H. R. Bourne, Proc. Natl. Acad. Sci. U.S.A. 104, 9296 (2007).

[26] A. Tamada, S. Kawase, F. Murakami, and H. Kamiguchi, J. Cell Biol. 188, 429 (2010).

[27] L. Q. Wan, K. Ronaldson, M. Park, G. Taylor, Y. Zhang, J. M. Gimble, and G. Vunjak-Novakovic, Proc. Natl. Acad. Sci. U.S.A. 108, 12295 (2011).

[28] C. L. Henley, J. Stat. Phys. 148, 741 (2012).

[29] S. Furthauer, M. Strempel, S. W. Grill, and F. Julicher, Phys. Rev. Lett. 110, 048103 (2013).

[30] S. R. Naganathan, S. Fürthauer, M. Nishikawa, F. Jülicher, and S. W. Grill, eLife 3, e04165 (2014).

[31] K. Taniguchi, R. Maeda, T. Ando, T. Okumura, N. Nakazawa, R. Hatori, M. Nakamura, S. Hozumi, H. Fujiwara, and K. Matsuno, Science 333, 339 (2011).

[32] R. Hatori, T. Ando, T. Sasamura, N. Nakazawa, M. Nakamura, K. Taniguchi, S. Hozumi, J. Kikuta, M. Ishii, and K. Matsuno, Mechanisms of development 133, 146 (2014).

[33] K. Sato, T. Hiraiwa, E. Maekara, A. Isomura, T. Shibata, and E. Kuranaga (unpublished). 